\title{
Normal and Abnormal Regulation of $\beta$-MSH in Man
}

\author{
Kaoru Abe, Wendell E. Nicholson, Grant W. Liddle, \\ David N. Orth, and Donald P. Island \\ From the Department of Medicine, Vanderbilt University School of Medicine, \\ Nashville, Tennessee 37203
}

A B S T R A C T The regulation of plasma $\beta$-melanocyte-stimulating hormone $(\beta-\mathrm{MSH})$ in man has been studied utilizing a radioimmunoassay previously described (1). In normal subjects plasma $\beta$-MSH values ranged from 20 to $110 \mathrm{pg} / \mathrm{ml}$. Metyrapone increased and dexamethasone decreased plasma $\beta$-MSH levels. Surgical stress stimulated $\beta$-MSH secretion. Plasma $\beta$-MSH levels were elevated in patients with untreated Addison's disease and untreated congenital adrenal hyperplasia, and these levels fell to normal during glucocorticoid therapy. In patients with Cushing's syndrome due to pituitary adrenocorticotropic hormone (ACTH) excess, plasma $\beta$-MSH was slightly elevated before treatment. In those patients who developed pituitary tumors and hyperpigmentation after bilateral adrenalectomy, plasma $\beta$-MSH was greatly elevated. In patients with Cushing's syndrome due to adrenal tumor, plasma $\beta$-MSH was subnormal. In patients with the ectopic ACTH syndrome, the levels of plasma $\beta$-MSH were high. Plasma $\beta$-MSH had a diurnal variation in normal subjects, patients with Addison's disease, and patients with congenital adrenal hyperplasia; but the normal diurnal variation was lost in patients with Cushing's disease. In patients with high plasma $\beta-\mathrm{MSH}$, simultaneous determinations of plasma ACTH showed close correlation between the degree of elevation of ACTH and that of $\beta$-MSH. In extracts of tumors from patients with the ectopic ACTH-MSH syndrome the quantities of the two hormones were roughly equivalent. In patients with hyperpigmentation due to a variety of disorders other than pituitary-adrenal abnormalities, plasma $\beta$-MSH was normal. It is concluded that the secretion of $\beta$-MSH is regulated by the same factors that regulate $\mathrm{ACTH}$.

\section{INTRODUCTION}

$\beta$-Melanocyte-stimulating hormone ( $\beta$-MSH) has previously been identified and quantified in the plasma of normal subjects and patients with addisonian hyperpigmen-

Received for publication 25 February 1969. tation by means of radioimmunoassay (1). On the basis of these studies it was concluded that $\beta-\mathrm{MSH}$ is probably the main pigmentary hormone in man and that the degree of addisonian hyperpigmentation correlates well with the degree of elevation of plasma $\beta$-MSH.

In the present study the same radioimmunoassay technique has been employed to clarify the regulation of $\beta$-MSH in normal subjects, patients with pituitaryadrenal abnormalities, patients with the ectopic adrenocorticotropic hormone (ACTH) syndrome, and patients with hyperpigmentation not associated with pituitary or adrenal diseases. In addition, $\beta-\mathrm{MSH}$ and ACTH were measured simultaneously in plasma samples and in tumors from patients with the ectopic ACTH syndrome to ascertain the quantitative relationship between these two hormones in this recently discovered disorder.

\section{METHODS}

Heparinized blood for $\beta$-MSH assay was collected by venipuncture, cooled immediately in ice, and the plasma was separated within minutes in a refrigerated centrifuge. The plasma was kept frozen on dry ice until extraction. Tissues were collected at surgery or autopsy, frozen, and stored on dry ice until extracted.

$\beta$-MSH was extracted from plasma or tissue and subjected to radioimmunoassay by a method described previously (1). Synthetic human $\beta-\mathrm{MSH}$ was used as a standard. ACTH was assayed in plasma by the method of Lipscomb and Nelson (2). Those samples of crude plasma in which ACTH was undetectable were extracted by the method of Island et al. (3) before assay. ACTH was extracted from tumors by the method of Island et al. (3) as subsequently modified for the extraction of $\beta$-MSH from tissue (1). Plasma 17,21-dihydroxy-20-ketosteroids (17-OHCS) were determined by a modification of the method of Silber and Porter (4).

\section{RESULTS}

Plasma $\beta-M S H$ levels in normal subjects. $\beta-\mathrm{MSH}$ was measured on 70 different occasions in 20 ambulatory normal subjects between the ages of 17 and $40 \mathrm{yr}$. The values ranged from 20 to $110 \mathrm{pg} / \mathrm{ml}$ with a mean of 50 and a SD of 23 . 


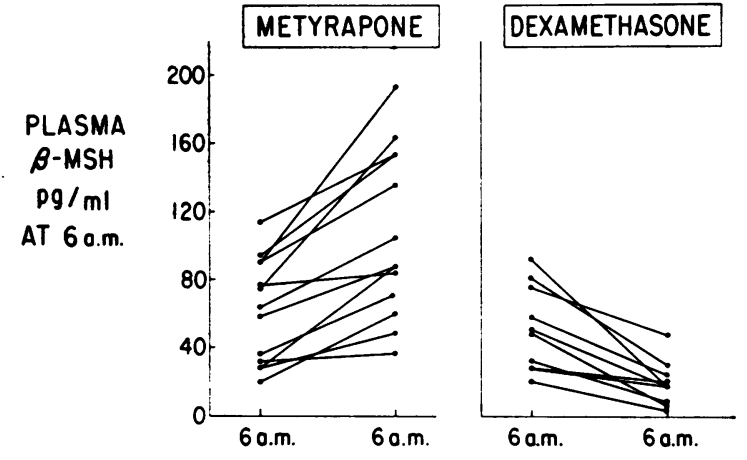

Figure 1 Left: Plasma $\beta$-MSH in normal subjects before and after treatment with metyrapone. Blood was drawn at 6 a.m. just before the first dose of metyrapone $(0.75 \mathrm{~g}$ every $4 \mathrm{hr}$ ) and again at $6 \mathrm{a} . \mathrm{m}$. on the following day. Right: Plasma $\beta$-MSH in normal subjects before and after treatment with dexamethasone. Blood was drawn at 6 a.m. before the first dose of dexamethasone ( $1 \mathrm{mg}$ every $6 \mathrm{hr}$ ) and again at 6 a.m. on the following day.

Metyrapone and dexamethasone tests. 13 normal subjects received $0.75 \mathrm{~g}$ of metyrapone orally every 4 $\mathrm{hr}$ for 1 day. Plasma was obtained for $\beta$-MSH assay just before the first dose of metyrapone at 6 a.m. and again at 6 a.m. on the following day. The results of this study are shown on the left in Fig. 1. In all cases treatment with metyrapone resulted in increased plasma $\boldsymbol{\beta}$-MSH levels.

10 other normal subjects were given $1 \mathrm{mg}$ of dexamethasone orally every $6 \mathrm{hr}$ for 1 day. Plasma was obtained for $\beta$-MSH assay at 6 a.m. on the day treatment was begun and 6 a.m. of the following day. The results are depicted on the right in Fig. 1. In each case plasma $\beta$-MSH was suppressed by dexamethasone, and in 7 of the 10 it was reduced to less than $20 \mathrm{pg} / \mathrm{ml}$.

Surgical stress. Plasma $\beta-\mathrm{MSH}$ was measured in eight patients undergoing hysterectomy. Blood was drawn for $\beta-\mathrm{MSH}$ and 17-OHCS measurements $1 \mathrm{hr}$ before surgery and again $1 \mathrm{hr}$ after the abdominal incision was made. The results of this study are depicted

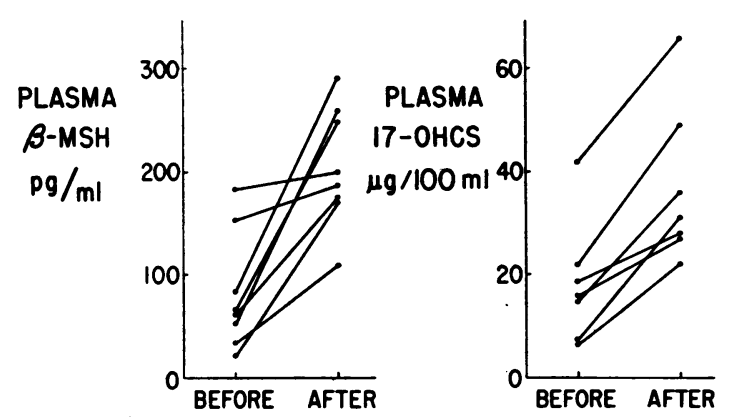

FIgURE 2 Plasma $\beta$-MSH in response to surgical stress. Blood was drawn for plasma $\beta-\mathrm{MSH}$ and $17-\mathrm{OHCS}$ assays $1 \mathrm{hr}$ before surgery and $1 \mathrm{hr}$ after the laparotomy incision.

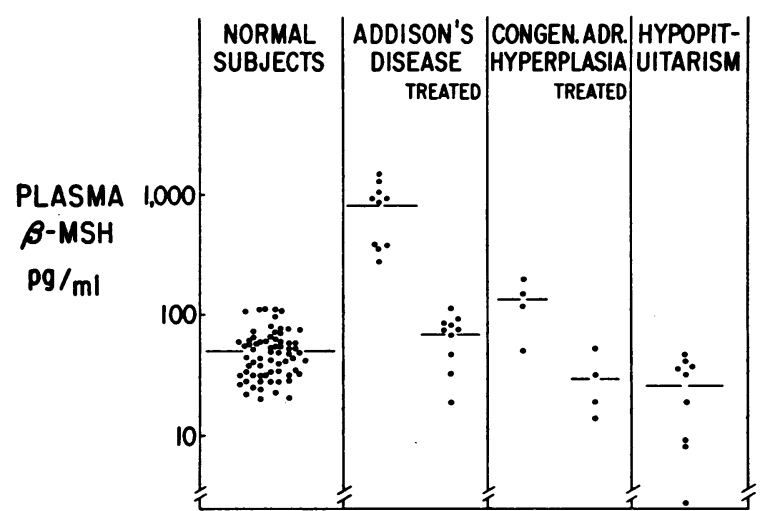

FIgURE 3 Plasma $\beta$-MSH in normal subjects and patients with Addison's disease, congenital adrenal hyperplasia, or hypopituitarism. The horizontal lines represent the means of various groups.

in Fig. 2. In all cases studied, plasma $\beta$-MSH and 17OHCS were found to rise during surgery. Two of the patients had plasma $\beta$-MSH levels above the normal range before surgery, and plasma $17-\mathrm{OHCS}$ were correspondingly higher in these two patients.

Addison's disease. Plasma $\beta$-MSH was measured in 10 cases of untreated Addison's disease. All values were greatly elevated, ranging from 266 to $1480 \mathrm{pg} / \mathrm{ml}$ with a mean value of $797 \mathrm{pg} / \mathrm{ml}$. In contrast, plasma $\beta$-MSH values in 10 well controlled addisonian patients were all within the normal range (Fig. 3 ).

Congenital adrenal hyperplasia. Plasma $\beta$-MSH was moderately elevated in three out of four patients with untreated congenital adrenal hyperplasia $(120-190 \mathrm{pg} /$ $\mathrm{ml})$; a normal value was found in the fourth. During treatment with corticosteroids, plasma $\beta$-MSH values were found to be low to normal (Fig. 3).

Hypopituitarism. In nine patients with hypopituitarism who required cortisol substitution therapy, plasma

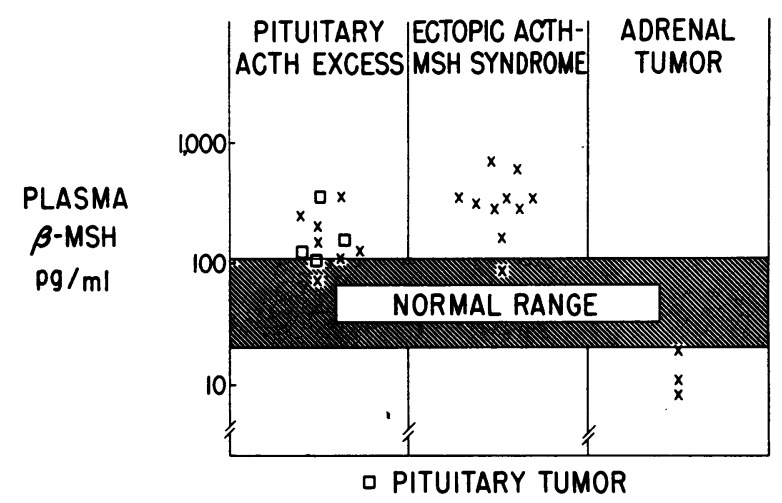

FIgUre 4 Plasma $\beta$-MSH in untreated Cushing's syndrome due to pituitary ACTH excess, ectopic ACTH-MSH syndrome, and adrenal tumor. 


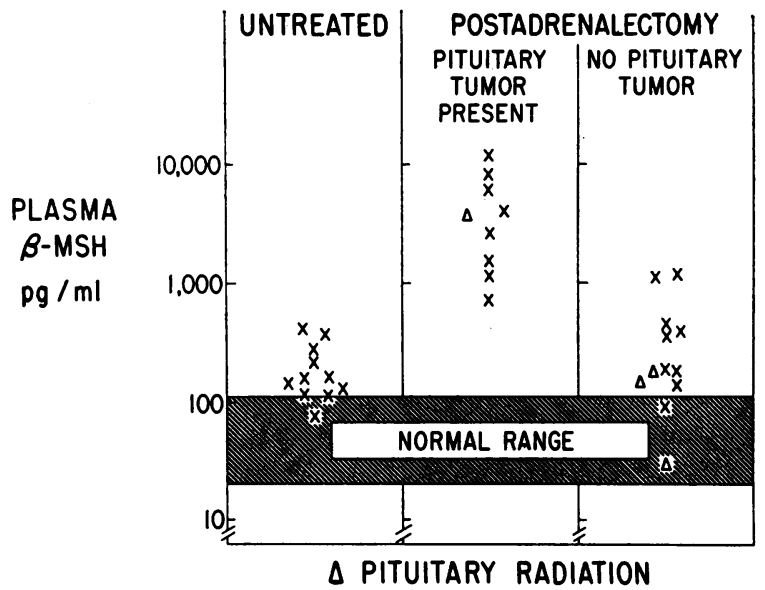

Figure 5 Plasma $\beta$-MSH in Cushing's disease (postadrenalectomy). For comparison, the data for untreated Cushing's disease in Fig. 4 are reproduced here. $\Delta=$ patients who had previously undergone pituitary irradiation.

$\beta$-MSH values were low, ranging from a negligible level to $46 \mathrm{pg} / \mathrm{ml}$ with a mean of $26 \mathrm{pg} / \mathrm{ml}$. Although there was some overlap between the values of normal subjects and those of patients with hypopituitarism, the group as a whole had plasma $\beta$-MSH values which were significantly lower than those in normal subjects $(P<0.01)$ (Fig. 3)

Cushing's syndrome due to pituitary ACTH excess. In 11 patients with untreated Cushing's syndrome due to pituitary ACTH excess (Cushing's disease), plasma $\beta$-MSH was moderately elevated. Four of these patients had enlargement of the sella turcica, but their plasma $\beta$-MSH values were in the same range as those of patients who did not have sellar enlargement (Fig. 4).

Cushing's syndrome due to ACTH production by a nonpituitary neoplasm (the ectopic ACTH-MSH syndrome). In 10 patients with untreated Cushing's syndrome due to ectopic $\mathrm{ACTH}$, plasma $\beta$-MSH was ele-

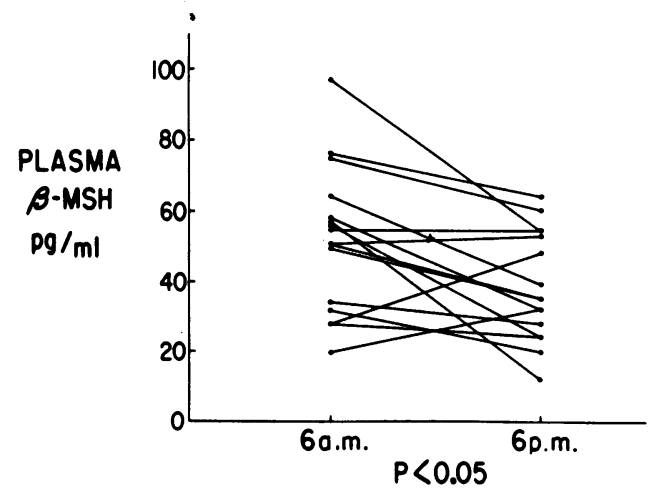

Figure 6 Diurnal variation of plasma $\beta$-MSH in normal subjects. Blood was drawn at 6 a.m. and 6 p.m. of the same day.

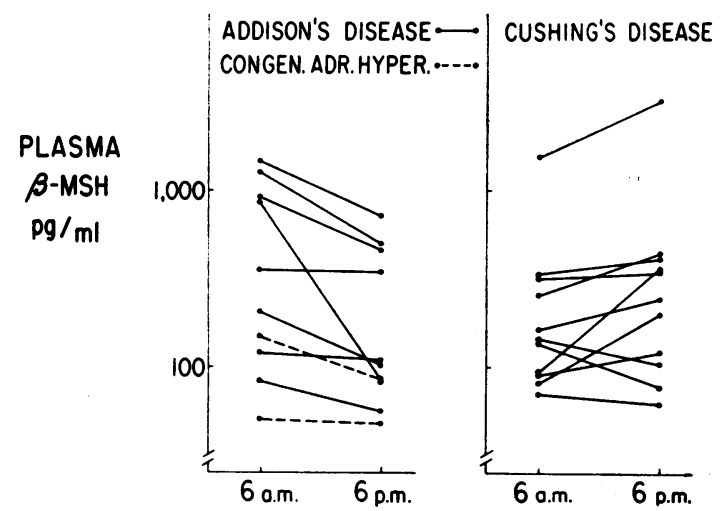

Figure 7 Diurnal variation of plasma $\beta$-MSH in Addison's disease, congenital adrenal hyperplasia, and Cushing's disease. Blood was drawn at 6 a.m. and 6 p.m. of the same day.

vated, ranging from 83 to $650 \mathrm{pg} / \mathrm{ml}$ with a mean of $341 \mathrm{pg} / \mathrm{ml}$ (Fig. 4). Three patients (not represented in Fig. 4) who had been subjected to bilateral adrenalectomy as treatment for their Cushing's syndrome had extremely high plasma $\beta$-MSH values $(900,1190$, and 5100 $\mathrm{pg} / \mathrm{ml}$ ) and were deeply pigmented.

Cushing's syndrome due to adrenal tumor. Three patients with untreated Cushing's syndrome due to adrenal tumors (one adenoma and two carcinomas) had subnormal levels of plasma $\beta$-MSH (Fig. 4).

Cushing's disease, postadrenalectomy. In nine patients who had developed pituitary tumors and hyperpigmentation after adrenalectomy as treatment for Cushing's disease, plasma $\beta-\mathrm{MSH}$ levels were extremely high, with a mean value of $4335 \mathrm{pg} / \mathrm{ml}$. In contrast, 12 patients who did not develop pituitary tumors after adrenalectomy for Cushing's disease had a mean plasma $\beta$-MSH value of $365 \mathrm{pg} / \mathrm{ml}$ (Fig. 5).

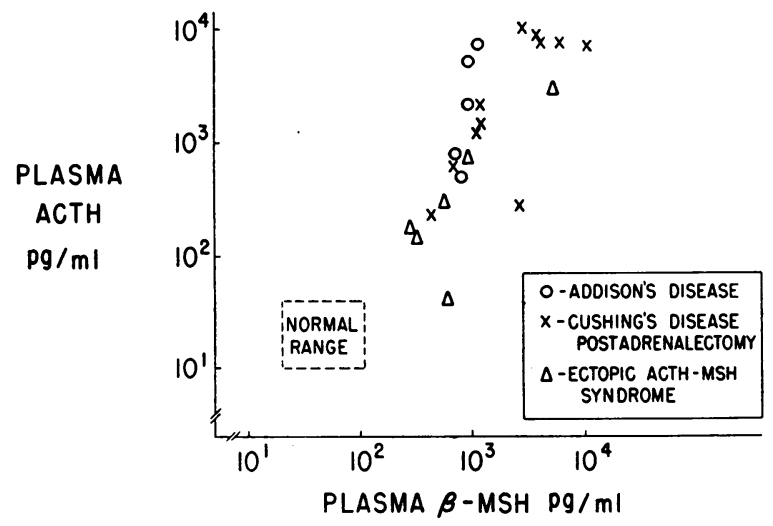

FIGURE 8 Correlation of plasma $\beta$-MSH and ACTH in Addison's disease, Cushing's disease (postadrenalectomy) and the ectopic ACTH-MSH syndrome. Highly purified human ACTH was used as a standard in the ACTH bioassay.

1582 K. Abe, W. E. Nicholson, G. W. Liddle, D. N. Orth, and D. P. Island 


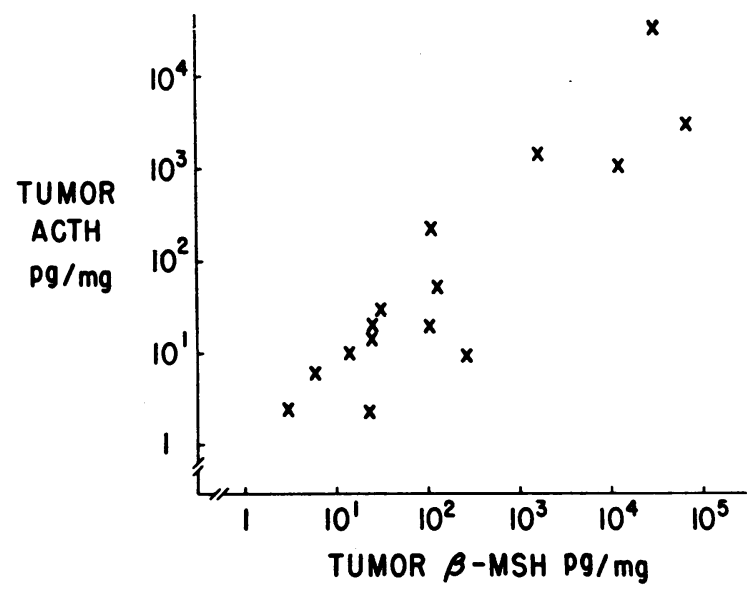

Figure 9 Correlation of $\beta$-MSH and ACTH in tumors from patients with the ectopic ACTH-MSH syndrome. $\mathrm{ACTH}$ and $\beta$-MSH are plotted in picograms per milligram of wet tissue. Highly purified human ACTH was used as a standard in the ACTH bioassay.

Diurnal variation of plasma $\beta-M S H$ in normal subjects. Diurnal variation of plasma $\beta$-MSH was examined in 16 normal subjects (Fig. 6). For the group as a whole, values were higher at 6 a.m. than 6 p.m. $(P<0.05)$.

Diurnal variation of plasma $\beta-M S H$ in Addison's disease, congenital adrenal hyperplasia, and Cushing's disease. In eight patients with Addison's disease, plasma $\beta$-MSH values were higher at 6 a.m. than at 6 p.m., and in two cases of congenital adrenal hyperplasia a similar diurnal variation was observed. For the 10 patients con-

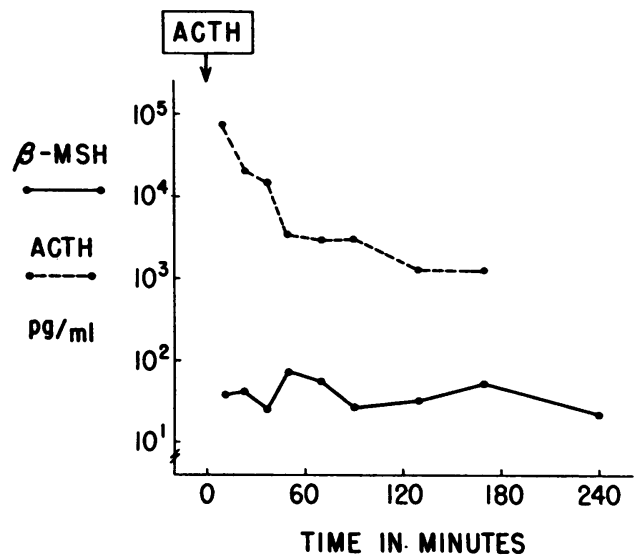

FIgURE 10 ACTH infusion study. $1 \mathrm{mg}$ of highly purified human ACTH was injected intravenously into a normal subject at time 0 . Blood was drawn from the opposite arm at the times indicated on the abscissa. The concentrations of $\beta$-MSH and ACTH found in the subject's plasma at various time intervals are plotted on the ordinate (log scale). A sample of the injected ACTH was used as a standard for the ACTH bioassay.
TABLE I

Pigmentary Disorders Associated with Normal Plasma $\beta$-MSH Levels

\begin{tabular}{cc}
\hline Disorder & $\begin{array}{c}\text { No. of } \\
\text { cases }\end{array}$ \\
\hline
\end{tabular}

A. Hyperpigmentation

Renal tubular acidosis $\quad 2$

Hyperthyroidism 3

Retinitis pigmentosa 2

Xeroderma pigmentosum 4

Liver cirrhosis 1

Hodgkin's disease 1

Etiology unknown

B. Albinism 7

sidered as a group, the difference was statistically significant $(P<0.05)$.

In contrast, most of the patients with Cushing's disease did not have a normal diurnal variation of plasma $\beta$-MSH (Fig. 7).

Correlation of plasma $\beta-M S H$ and ACTH. Plasma $\beta$-MSH and ACTH were measured in the same plasma samples from five patients with Addison's disease, 11 patients who had undergone bilateral adrenalectomy as treatment for Cushing's disease, and six patients with the ectopic ACTH-MSH syndrome. As shown in Fig. 8 , significant positive correlation existed between the plasma levels of $\beta$-MSH and ACTH, $(r=0.70, P<$ 0.001 ).

Correlation of $\beta-M S H$ and $A C T H$ in tumors from patients with the ectopic ACTH-MSH syndrome. $\beta$-MSH and ACTH were measured in the same extracts of tumors from 15 patients with the ectopic ACTHMSH syndrome. As indicated in Fig. 9, a high degree of positive correlation existed between the concentrations of $\beta-\mathrm{MSH}$ and ACTH in these tumors $(r=0.91$, $P<0.001)$.

Hyperpigmentation associated with disorders other than pituitary-adrenal abnormalities. Patients with hyperpigmentation associated with renal tubular acidosis, hyperthyroidism, retinitis pigmentosa, xeroderma pigmentosum, hepatic cirrhosis, and Hodgkin's disease had normal plasma $\beta$-MSH levels. In addition, seven albinos had normal plasma $\beta$-MSH (Table I). No racial differences in plasma $\beta$-MSH levels were observed among Caucasian, Negro, and Japanese subjects.

$A C T H$ infusion study. Even though pure ACTH does not interfere with the assay of $\beta-\mathrm{MSH}$ (1), the theoretical possibility existed that the $\beta$-MSH which was measured by this radioimmunoassay technique might represent metabolized ACTH. To exclude this possibility the following experiment was done. $1 \mathrm{mg}$ of highly purified human ACTH was injected intravenously into 
a normal subject. As shown in Fig. 10, although plasma ACTH reached a concentration of $71,000 \mathrm{pg} / \mathrm{ml}$ and during the next $170 \mathrm{~min}$ gradually fell to $1220 \mathrm{pg} / \mathrm{ml}$, plasma $\beta$-MSH levels remained within the normal range. It was concluded that ACTH metabolites do not interfere to a significant degree with the determination of $\beta$-MSH by the method employed in this study.

\section{DISCUSSION}

The secretion of $\beta$-MSH by the normal human pituitary appears to be regulated by three major factors. First, glucocorticoids have a suppressive influence on $\beta-\mathrm{MSH}$ secretion. Thus, the administration of the synthetic glucocorticoid, dexamethasone, to normal subjects causes a decrease in plasma $\beta$-MSH, and the hypersecretion of cortisol by an autonomous adrenocortical neoplasm is associated with subnormal plasma levels of $\beta$-MSH. On the other hand, impaired secretion of cortisol (due to Addison's disease, congenitial adrenal hyperplasia, or treatment with metyrapone) leads to an increase in plasma $\beta-\mathrm{MSH}$; and this increase can be reversed by treatment with physiologic doses of cortisol or other glucocorticoids. Second, there is a factor that confers diurnal rhythmicity on $\beta$-MSH secretion; as a consequence, $\beta$-MSH concentrations in the plasma are higher in the morning than in the evening. Third, stress such as that associated with a laparotomy causes increased secretion of $\beta$-MSH. It thus appears that the physiologic regulation of $\beta-\mathrm{MSH}$ is under the influence of the same factors that control the secretion of ACTH (5): glucocorticoids, diurnal rhythm, and stress.

In addition to the conditions mentioned above, in which changes in $\beta$-MSH and ACTH secretion occur as appropriate responses by the normal pituitary to extrapituitary factors, there are a number of pathologic states in which there appears to be a primary disturbance of $\beta$-MSH (and ACTH) secretion. In hypopituitarism, despite subnormal glucocorticoid levels, $\beta-\mathrm{MSH}$ levels are subnormal. In Cushing's disease, despite supernormal cortisol levels, $\beta$-MSH levels are usually supernormal and lack the normal diurnal rhythm. In patients with the ectopic ACTH syndrome, nonpituitary tumors secrete $\beta$-MSH and give rise to supernormal plasma concentrations of this hormone. In all of these situations, alterations in plasma $\beta-\mathrm{MSH}$ tend to parallel alterations in plasma ACTH.

Since $\beta$-MSH appears to be a constant companion of endogenous $\mathrm{ACTH}$, the question has been raised as to whether immunoreactive " $\beta$-MSH" might merely be a degradation product of ACTH. This has been answered in the negative, however, in experiments in which very large quantities of pure ACTH were infused intravenously, giving rise to extreme elevations of plasma $\mathrm{ACTH}$; neither the appearance of pure exogenous
$\mathrm{ACTH}$ in the circulation nor its disappearance from the circulation resulted in appreciable increases in plasma immunoreactive $\beta$-MSH.

Our data are consistent with the view that $\beta-\mathrm{MSH}$ is the principal pigmentary hormone in man. Although ACTH has intrinsic melanotropic activity, it is only about $4 \%$ as active as $\beta$-MSH itself (6). Since the concentrations of ACTH and $\beta$-MSH are approximately equal in plasma, it is evident that ACTH does not contribute a major portion of the melanotropic activity of plasma. The concentrations of $\alpha-\mathrm{MSH}$ have thus far been too low to measure by radioimmunoassay. Furthermore, if one performs bioassays and radioimmunoassays on the same plasma extracts, one can usually account for most of the biological MSH activity by the quantity of $\beta$-MSH measured by radioimmunoassay (1). Thus, all of the available data support the view that $\beta$-MSH is the principal melanocyte-stimulating factor in human plasma. This conclusion requires the assumption that the ratios of potency for these hormones are the same in man as in the frog, even though the qualitative character of the melanocyte response may be different in the two species.

Evidence has been presented previously (1), and again in this study, that the degree of chronic elevation of circulating $\beta-\mathrm{MSH}$ is proportional to the degree of hyperpigmentation exhibited by affected patients. Pronounced hyperpigmentation attributable to $\beta$-MSH is encountered in some patients with untreated Addison's disease, some who have undergone bilateral adrenalectomy for Cushing's disease, and some who have been adrenalectomized as treatment for the "ectopic ACTHMSH syndrome." These disorders are to be distinguished from other states of hyperpigmentation in which $\beta$-MSH concentrations are quite normal; these include the hyperpigmentation occasionally seen in renal tubular acidosis, hyperthyroidism, xeroderma pigmentosum, hepatic cirrhosis, and Hodgkin's disease.

The teleologic importance (if any) of $\beta-\mathrm{MSH}$ in human physiology is unknown. Albinos, whose lack of pigmentation might (hypothetically) call forth increased secretion of a teleologically useful pigmentary hormone, have been found to have only normal plasma concentrations of $\beta$-MSH. Nor have there been racial differences in $\beta$-MSH that would fit either a teleological pattern or provide an explanation for differences in racial pigmentation.

It can be concluded that $\beta$-MSH production is closely linked to ACTH production in health and disease. A1though the hyperpigmentation caused by extremely high concentrations of $\beta$-MSH can be of diagnostic utility and is sometimes a cosmetic problem to the patient, the possible physiological importance of $\beta-\mathrm{MSH}$ in man remains unknown. 


\section{ACKNOWLEDGMENTS}

Synthetic human $\beta$-MSH was kindly provided by Doctors H. Bein, P. DeSaulles, and W. Rittel of CIBA, Ltd., Basle, Switzerland and highly purified human ACTH by Doctors G. V. Upton and A. B. Lerner of Yale University.

These studies were supported in part by Grants-in-Aid 5-K6-AM-3782, 8-MO1-FR-95, 5-TO1-AM-05092, and 2RO1-AM-05318 from the National Institutes of Health, U. S. Public Health Service.

\section{REFERENCES}

1. Abe, K., W. E. Nicholson, G. W. Liddle, D. P. Island, and D. N. Orth. 1967. Radioimmunoassay of $\beta$-MSH in human plasma and tissues. J. Clin. Invest. 46: 1609.
2. Lipscomb, H. S., and D. H. Nelson. A sensitive biologic assay for ACTH. 1962. Endocrinology. 71: 13.

3. Island, D. P., N. Shimizu, W. E. Nicholson, K. Abe, E. Ogata, and G. W. Liddle. 1965. A method for separating small quantities of $\mathrm{MSH}$ and $\mathrm{ACTH}$ with good recovery of each. J. Clin. Endocrinol. 25: 975.

4. Silber, R. H., and C. C. Porter. 1954. The determination of 17,21-dihydroxy-20-ketosteroids in urine and plasma. J. Biol. Chem. 210: 923.

5. Liddle, G. W., D. P. Island, and C. K. Meador. 1962. Normal and abnormal regulation of corticotropin secretion in man. Recent Progr. Hormone Res. 18: 125.

6. Ney, R. L., E. Ogata, N. Shimizu, W. E. Nicholson, and G. W. Liddle. 1964. Structure-Function relationships of ACTH and MSH analogues. Excerpta Med. Int. Congr. Ser. 83: 1184. 